\title{
Can PCI Ever Replace Optimal Medical Therapy in Treating "Stable" Epicardial Coronary Stenosis?
}

\section{Cheuk-Kit Wong}

Department of Medicine and Therapeutics, Faculty of Medicine, The Chinese University of Hong Kong, Hong Kong

*Corresponding author: Cheuk-Kit Wong, Department of Medicine and Therapeutics, Faculty of Medicine, The Chinese University of Hong Kong, Prince of Wales Hospital Shatin, Hong Kong, Tel: 852-35053139; E-mail: cheuk-kit.wong@cuhk.edu.hk

Received date: November 23, 2017; Accepted date: November 27, 2017; Published date: December 01, 2017

Copyright: (c) 2017 Wong CK, This is an open-access article distributed under the terms of the Creative Commons Attribution License, which permits unrestricted use, distribution, and reproduction in any medium, provided the original author and source are credited.

\section{Editorial}

In 2007, the COURAGE trial was published showing that in the 2287 randomized patients with stable coronary disease PCI was no better than medical therapy in altering outcome over a median followup period of 4.6 years [1]. The same mortality rate observed among the 2 randomized groups held true at late median follow-up of 6.2 years amongst the 1211 patients with available survival data [2]. One major criticism of COURAGE is that PCI (including the nature of the deployed stents and adjunctive pharmacological therapy) has evolved so rapidly in the last 10 years that the COURAGE findings are no longer applicable today. Modern drug eluting stents plus appropriately potent antiplatelet regimes have ensured almost $100 \%$ procedural successes with $<1 \%$ stent thrombosis and low restenosis rates. Prior editorials in the Journal have discussed some of these aspects [3-6]. It is tempting for one to think that PCI with stenting will fix any stable epicardial coronary stenosis relieving myocardial ischemia with measurable clinical benefit. However, 2 recent trials have reported somewhat unexpected results.

In the ORBITA trial [7], a double-blinded, multicentre randomised trial of real PCI versus "sham PCI" for angina relief at 5 UK study sites, symptomatic patients with severe ( $\geq 70 \%$ diameter narrowing) singlevessel stenosis were enrolled. They first received 6 weeks of medical therapy optimisation followed by cardiopulmonary exercise testing, symptom questionnaires, and dobutamine stress echocardiography. Then, they were randomised 1:1 to undergo PCI or a placebo procedure. In both groups fractional flow reserve (FFR) and instantaneous flow reserve (iFR) were measured. These FFR and iFR measurements required introducing special coronary wires across the coronary lesion so all patients actually needed the cath lab procedures. Patients were sufficiently sedated together with "auditory isolation" that they were unaware as to whether they received real or sham PCI. Six weeks after the procedure the aforementioned non-invasive assessments were repeated. The primary endpoint was difference in exercise time increment between groups.

Of 230 patients enrolled, 200 were finally randomised in the period from January 2014 to August 2017 after completing the medication optimisation phase. The coronary lesions had mean area stenosis of $84.4 \%$, FFR of 0.69 (FFR $<0.75-0.8$ generally considered significant indicating myocardial ischemia in the subtended territory), and iFR of 0.76 (iFR < 0.9 generally considered significant). All PCI procedures were successful but the primary endpoint, exercise time increment between groups (PCI minus placebo), was only non-significantly higher by 16.6 seconds $(\mathrm{P}=0.20)$.

Intervention trials with "clinical procedures" are almost always confounded because of the absence of patient blinding unless sham procedures are performed in the control group with all their implications. In the hypertension field, there had been high hopes for renal denervation until it was refuted by the sham-procedure controlled SYMPLICITY HTN-3 trial [8]. ORBITA has reached the highest standard for randomised controlled trial because it was double-blinded so that even the managing team did not know the randomized treatment patients received. Patients had medically welltreated angina before PCI as reflected by their relatively good performance on exercise test despite having severe coronary stenoses causing ischemia as reflected by $\mathrm{FFR} / \mathrm{iFR}$ and dobutamine stress echocardiography findings.

The salient finding in ORBITA is that PCI did not increase exercise time more than that from sham procedure. This stands in contrast to many anti-anginal drugs where therapy significantly prolongs exercise time in placebo-controlled randomised trials. It should be noted that current guidelines have generally supported the use of PCI in ORBITA patients as they had both objective evidence of ischemia and anginal symptoms.

Unlike COURAGE [1,2], ORBITA [7] is far too small for clinical outcome end-points. In fact, stable single-vessel coronary disease medically managed today is having such good survival that showing even small mortality advantage will require large patient numbers. How then can we establish whether PCI with stenting on stable lesion(s) improves survival? The currently on-going ISCHEMIA trial addresses this question in thousands of patients including many with multi-vessel disease but results will not be available until some years later. However, indirect tangential evidence has emerged from the recent CULPRIT-SHOCK trial [9].

In CULPRIT-SHOCK [9], 706 patients with multi-vessel disease, acute myocardial infarction (AMI), and cardiogenic shock were randomized to PCI of the culprit lesion only (with potentially staged PCI for non-culprit lesions) or to immediate multi-vessel PCI (including non-culprit artery lesions) in the period from April 2013 to April 2017. Non-culprit artery lesions may be regarded, with some caveats, as having pathologic characteristics largely resembling lesions found in patients with stable angina.

Data were analysed by the Intention-to-treat analysis. Cross-over rate was $\sim 10 \%$ in both randomized groups. Staged revascularization was performed in 61 of the 344 patients in the culprit-lesion-only PCI group. At 30 days, the composite primary end point (death or renalreplacement therapy) was lower in the culprit-lesion-only PCI group than in the multi-vessel PCI group (45.9\% vs. 55.4\%, P=0.01). Death occurred less frequently in the culprit-lesion-only PCI group than in the multi-vessel PCI group (43.3\% vs. 51.6\%; $\mathrm{P}=0.03)$, with the survival curves separating from Day 5 onwards. There was no difference between the 2 groups with respect to the time to hemodynamic 
stabilization, the use and duration of catecholamine therapy, the levels of troponin $\mathrm{T}$ and creatine kinase, and the rates of bleeding and stroke.

The CULPRIT-SHOCK findings are somewhat unexpected as multivessel PCI for AMI complicated by cardiogenic shock is currently supported by guidelines or appropriate-use criteria in most parts of the world including Europe and America. The main rationale for this multi-vessel PCI strategy is that cardiogenic shock represents the most difficult time for the struggling ischemic myocardium so that perfusion to all ischemic area including those subtended by non-infarct arteries should be fully achieved, thus the additional PCI for angiographically significant non-infarct artery lesions.

The CULPRIT-SHOCK trial confirmed the high 30-day mortality of the dreadful combination of AMI, cardiogenic shock, and multi-vessel disease. Mortality is the most concrete end-point in clinical trials and the most relevant endpoint in high risk cardiac situations. To explain the higher mortality and poorer outcome with additional non-infarct artery PCI in CULPRIT-SHOCK, the authors speculated that the acute hazards from prolonging PCI procedure using more radiographic contrast had outweighed any potential benefit from the multi-vessel PCI. However, there is potentially another intriguing explanation that requires more detailed explanation.

Given that in CULPRIT-SHOCK trial only $\sim 17 \%$ of the patients had prior MI, $\sim 19 \%$ had prior PCI and $\sim 5 \%$ had prior coronary artery bypass grafting, many without known coronary disease were likely not taking statins prior to their acute presentations. Such data were not available in their current report [9]. Taking high dose statins before PCI (as compared to no statins or low dose statins) has been shown to reduce peri-PCI infarctions and 30 -day events by $\sim 50 \%$ in a metaanalysis of 13 studies with 3341 patients [10]. The benefit was present irrespective of clinical presentations and subgroups, but appeared greater in those with higher baseline C-reactive protein (CRP) levels [10].

Distal plaque embolization occurs quite commonly during PCI. The extent of embolization is related to the plaque volume and plaque composition. The ventricular impairment secondary to this embolization (causing the peri-PCI infarction described above) during non-culprit artery PCI is likely more severe and haemodynamically relevant in the already compromised patients with on-going cardiogenic shock, as in the CULPRIT-SHOCK trial patients.

Using virtual histology intravascular ultrasound imaging which allows real-time determination of plaque composition in-vivo from radiofrequency analysis of the backscattered ultrasound signal, it is found that plaques with thin cap (so-called thin-capped fibroatheroma) and large necrotic core representing fatty/lipid substance are associated with more distal embolization and ischemia during PCI [11]. Statins stabilize coronary plaque reducing plaque size, altering plaque constituents and thickening the fibrous cap [12], as well as lower CRP. Of interest, plaque stabilization has been found to be more pronounced in those with greater reduction of CRP level [13]. CRP can be measured by high sensitive analysis (hs-CRP) reflecting the intensity of inflammation from the atherosclerotic process.

In patients with stable angina, inflammation with high hs-CRP level portends worse outcome. This may be reversed by statins and as found recently in the CANTOS trial [14] by canakinumab, a monoclonal antibody targeting interleukin-1 $\beta$. CANTOS randomized in a doubleblind manner 10061 patients with previous MI and hs-CRP level >2 $\mathrm{mg} / \mathrm{liter}$. The trial compared three doses of canakinumab (50 mg, 150 $\mathrm{mg}$, and $300 \mathrm{mg}$ ) administered subcutaneously every 3 months with placebo. Canakinumab reduced hs-CRP level in a dose-related manner but it did not reduce lipid levels. At a median follow-up of 3.7 years, the incidence rate for the primary efficacy end point (nonfatal MI, nonfatal stroke, or cardiovascular death) was lower with canakinumab. The hazard ratios as compared with placebo were 0.93 with $50 \mathrm{mg}$ $(\mathrm{P}=0.30) ; 0.85$ with $150 \mathrm{mg}(\mathrm{P}=0.021)$; and 0.86 with $300 \mathrm{mg}$ $(\mathrm{P}=0.031)$. All-cause mortality was not different (hazard ratio for all canakinumab doses vs. placebo 0.94; $\mathrm{P}=0.31$ ). Exploratory analysis showed that the clinical benefit of canakinumab was most profound in those who had a mark drop in their hs-CRP level after treatment [15].

The role of high-dose statins (+/- ezetimide) in lowering LDL level in patients with vascular disease is universally accepted. Positive results in improving outcome are emerging for PCSK9 inhibitors. While PCI and medical therapy have often been portrayed as competing treatment options, they are actually complementary in many aspects. Stent thrombosis is reduced by appropriate regime of anti-platelet drugs [3-6]. Peri-PCI embolization/infarction is reduced by high-dose statins [10] and possibly by canakinumab which helps stabilizing plaques.

The ORBITA findings [7] show that with very well optimised medical therapy PCI offers little additional symptomatic benefit. The CULPRIT-SHOCK findings serve to illustrate that PCI on stable noninfarct related lesions is not without its own risks [9]. The risk includes peri-PCI plaque embolization which may occur more frequently in patients without prior statin therapy. Perhaps the debate "Can PCI ever replaces optimal medical therapy in treating "stable" epicardial coronary stenosis?" should now be over. The new focus is on how we optimally combine these 2 effective strategies in managing patients.

\section{References}

1. Boden WE, O'Rourke RA, Teo KK (2007) COURAGE Trial Research Group. Optimal medical therapy with or without PCI for stable coronary disease. N Engl J Med 356: 1503-1516.

2. Sedlis SP, Hartigan PM, Teo KK (2015) COURAGE Trial Investigators. Effect of PCI on Long-Term Survival in Patients with Stable Ischemic Heart Disease. N Engl J Med 373: 1937-1946.

3. Wong CK (2012) Measuring Platelet Reactivity after Clopidogrel-Has it Reached the End of the Road. Cardiol Pharmacol 1: e105.

4. Wong CK (2016) From Arctic to Antarctic: Is There any Remaining Role for Platelet Function Testing to Tailor Therapy after Coronary Stent Implantation. Cardiovasc Pharm Open Access 5: e132.

5. Wong CK (2017) Trials Evaluating Ticagrelor in Cardiovascular Disease: Will It Reign Supreme in the Anti-Platelet World. Cardiovasc Pharm Open Access 6: e133.

6. Wong CK (2017) Tailoring Dual Antiplatelet Therapy after Coronary Stenting: The PRECISE-DAPT Score versus the DAPT Score. Cardiovasc Pharm Open Access 6: e135.

7. Al-Lamee R, Thompson D, Dehbi HM (2017) ORBITA investigators. Percutaneous coronary intervention in stable angina (ORBITA): a doubleblind, randomised controlled trial. Lancet 17: 32714-32719.

8. Bhatt DL, Kandzari DE, O'Neill WW (2014) SYMPLICITY HTN-3 Investigators. A controlled trial of renal denervation for resistant hypertension. N Engl J Med 370: 1393-1401.

9. Thiele H, Akin I, Sandri M (2017) CULPRIT-SHOCK Investigators. PCI strategies in patients with Acute Myocardial Infarction and Cardiogenic Shock. N Engl J Med 30.

10. Patti G, Cannon CP, Murphy SA (2011) Clinical benefit of statin pretreatment in patients undergoing percutaneous coronary intervention: a collaborative patient-level meta-analysis of 13 randomized studies. Circulation 123: 1622-1632. 
Citation: Wong CK (2017) Can PCI Ever Replace Optimal Medical Therapy in Treating "Stable" Epicardial Coronary Stenosis?. Cardiovasc Pharm Open Access 6: e137. doi:10.4172/2329-6607.1000e137

Page 3 of 3

11. Claessen BE, Maehara A, Fahy M, Xu K, Stone GW, et al. (2012) Plaque composition by intravascular ultrasound and distal embolization after percutaneous coronary intervention. JACC Cardiovasc Imaging 5: S111-118.

12. Min JK, Chandrashekhar Y, Narula J (2017) The Immediate Effects of Statins on Coronary Atherosclerosis: Can Phenotype Explain Outcome. JACC Cardiovasc Imaging 9.

13. Koskinas KC, Zaugg S, Yamaji K (2016) Changes of coronary plaque composition correlate with C-reactive protein levels in patients with ST- elevation myocardial infarction following high-intensity statin therapy. Atherosclerosis 247: 154-160.

14. Ridker PM, Everett BM, Thuren T (2017) CANTOS Trial Group. Antiinflammatory Therapy with Canakinumab for Atherosclerotic Disease. N Engl J Med 377: 1119-1131.

15. Ridker PM, MacFadyen JG, Everett BM (2017) CANTOS Trial Group. Relationship of C-reactive protein reduction to cardiovascular event reduction following treatment with canakinumab: a secondary analysis from the CANTOS randomised controlled trial. Lancet. 